\title{
Aplicação de modelos de regressão aleatória utilizando diferentes estruturas de dados
}

\author{
Application of random regression models using different data structures
}

\author{
Severino Cavalcante de Sousa Júnior ${ }^{*}$ Lenira El Faro ${ }^{\mathrm{II}}$ Annaiza Braga Bignardi ${ }^{\mathrm{I}}$ \\ Vera Lucia Cardoso ${ }^{\text {II }}$ Paulo Fernando Machado ${ }^{\text {III }}$ Lucia Galvão de Albuquerque ${ }^{\mathrm{I}}$
}

RESUMO

Foram utilizadas 3.202 primeiras lactações de vacas da raça Holandesa de quatro fazendas da região Sudeste, para verificar a influência da estrutura de dados de produção de leite sobre os parâmetros genéticos. Foram testados quatro arquivos com diferentes estruturas: controles semanais (CS), arquivo mensal $(C M)$, bimestral (CB) e trimestral $(C T)$, com 122.842, 30.883, 15.837 e 12.702 controles, respectivamente. Um modelo de regressão aleatória foi empregado nas análises, considerando os efeitos genético aditivo e o de ambiente permanente de animal, como aleatórios. Os efeitos fixos, grupos de contemporâneos $(G C)$ foram comuns para todos os arquivos de dados e foram compostos por fazenda, mês e ano do controle, além da co-variável idade da vaca ao parto (regressão linear e quadrática). As estimativas de herdabilidade apresentaram tendências mais semelhantes entre os arquivos de dados CS, CM e CB. O arquivo de dados CB apresentou estimativas de parâmetros genéticos com as mesmas tendências e magnitudes que os arquivos CS e CM, permitindo afirmar que não houve influência da estrutura dos dados sobre as estimativas dos componentes de (co)variância e que o controle leiteiro poderia ser realizado em uma estrutura $C B$.

Palavras-chave: avaliação genética, bovinos de leite, dados longitudinais.

\section{ABSTRACT}

A total of 3.035 lactations of Holstein cows from four farms in the Southeast, to check the influence of data structure of milk yield on the genetic parameters. Four dataset with different structures were tested, weekly controls $(C W)$ with 122.842 controls, monthly controls (CM) 30.883, bimonthly controls (CB) with 15,837 and quarterly controls $(C Q)$ with 12,702. The random regression model was used and was considered as random additive genetic and permanent environment effects, fixed effects of the contemporary groups (herd-year-month of test-day) and age of

\begin{abstract}
cow (linear and quadratic effects). Heritability estimates showed similar trends among the data files analyzed, with the greatest similarity between dataset $C S, C M$ and $C B$. The dataset submitted all the $C B$ estimates of genetic parameters analyzed with the same trend and similar magnitude to the CS and CM dataset, allowing the claim that there was no influence of the data structure on estimates of covariance components for the dataset CS, CM and $C B$. Thus, milk recording could be accomplished in a CB structure.
\end{abstract}

Key words: dairy cattle, genetic evaluation, longitudinal data.

\section{INTRODUÇÃO}

Os modelos de regressão aleatória (MRA) têm se tornado uma alternativa padrão para análises genéticas de dados longitudinais. Tais modelos têm sido mais utilizados para avaliações genéticas de bovinos leiteiros, empregando a produção de leite no dia do controle (Schaeffer, 2004).

Os MRA apresentam a vantagem de ajustar as produções de leite em cada controle, permitindo descrever os efeitos ambientais específicos ao controle, além de possibilitar a estimação de curvas de lactação genéticas para cada animal, como desvio da curva de lactação média. Um dos entraves dos MRA está relacionado à exigência de memória e tempo computacional para a realização de avaliações genéticas em larga escala. Assim, alternativas têm sido propostas para diminuir o número de parâmetros estimados, como o uso de funções de variâncias para modelar a estrutura de variâncias residuais e

\footnotetext{
IDepartamento de Zootecnia, Universidade Estadual Júlio de Mesquita (UNESP), Via de acesso Prof. Paulo Donato Castellane, s/n, 14884-900, Jaboticabal, SP, Brasil. E-mail: sevzoo@yahoo.com.br. *Autor para correspondência.

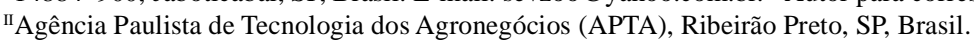

"IIEscola Superior de Agricultura "Luiz de Queiroz" (ESALQ), Universidade de São Paulo (USP), Piracicaba, SP, Brasil Recebido 10.08.13 Aprovado 25.02.14 Devolvido pelo autor 28.07.14 CR-2013-1082.R2
} 
funções de correlações paramétricas para descrever a estrutura de correlações de ambiente permanente devido ao animal (MEYER, 2001; ALBUQUERQUE \& MEYER, 2005).

A estrutura dos dados deve, certamente, exercer grande influência na estimação dos parâmetros genéticos obtidos pelos MRA (MEYER, 1998a). Estudos de gado de leite e corte encontraram maiores herdabilidades nos pontos extremos da lactação (MELO et al, 2000; EL FARO \& ALBUQUERQUE, 2005; BIGNARDI et al., 2009a), sugerindo que a menor quantidade de informações neste período influenciava as estimativas. Além disso, alguns trabalhos têm constatado correlações genéticas negativas entre as produções do início e do final da lactação (MEYER, 1998b; EL FARO et al., 2003; BIGNARDI et al., 2009a,b).

O objetivo do presente estudo foi avaliar a influência de diferentes estruturas de dados sobre as estimativas de parâmetros genéticos para a produção de leite no dia do controle, aplicando-se modelos de regressão aleatória.

\section{MATERIAL E MÉTODOS}

Foram utilizadas 3.202 primeiras lactações de vacas da raça Holandesa, provenientes de quatro rebanhos da região Sudeste do Brasil, com partos registrados no período de 1995 a 2003. As propriedades possuíam controles leiteiros realizados semanalmente ou diariamente. Dessa forma, foi utilizado um banco de dados em que todos os animais foram controlados semanalmente, que foi considerado o arquivo completo (CS). A partir deste, foram criados diferentes subconjuntos de dados quanto à frequência do controle leiteiro, para verificar a influência da estrutura dos dados sobre a estimação dos parâmetros genéticos para a produção de leite: a) Controle semanal (CS): todos os animais foram controlados semanalmente da $1^{\mathrm{a}}$ até a $43^{\mathrm{a}}$ semana de lactação (305 dias). Esta foi considerada a situação referência que serviu de base de comparação para as demais estruturas. b) Controle mensal (CM): todos os animais foram controlados mensalmente do $1^{\circ}$ ao $10^{\circ}$ mês da lactação; c) Controle bimestral (CB): todos os animais foram controlados bimestralmente, originando cinco classes de controles por animal, abrangendo os seguintes meses de lactação: $1^{\circ}, 3^{\circ}, 5^{\circ}$, $7^{\circ}, 10^{\circ} ;$ d) Controle trimestral (CT): todos os animais foram controlados trimestralmente, originando quatro classes de controles, abrangendo os seguintes meses de lactação: $1^{\circ}, 4^{\circ}, 7^{\circ}, 10^{\circ}$.

As análises foram realizadas por meio de um MRA uni-característica, tendo como variável resposta a produção de leite em cada controle (PLDC), sendo incluídos, como efeitos aleatórios, o genético aditivo e de ambiente permanente direto e o residual. Os efeitos fixos foram os grupos de contemporâneos, definidos por fazenda, mês e ano do controle, além da idade da vaca ao parto como covariável (efeito linear e quadrático) e a curva média de lactação da população, esta modelada por meio de um polinômio ortogonal de quarta ordem. Os efeitos aleatórios genéticos aditivos e de ambiente permanente foram modelados por meio de regressão aleatória sobre polinômios ortogonais de Legendre. O MRA pode ser descrito por:

$$
y_{i j}=F+\sum_{m=0}^{k b-1} \beta_{m} \phi_{m}\left(t_{i}\right)+\sum_{m=0}^{k a-1} a_{j m} \phi_{m}\left(t_{i j}\right)+\sum_{m=0}^{k p-1} \gamma_{j m} \phi_{m}\left(t_{i j}\right)+E_{i j}
$$

Em que, $y i j=$ produção de leite do j-ésimo animal, no i- ${ }^{-}$simo controle; $F=$ conjunto de efeitos fixos GC e idade ao parto; $\beta_{m}=$ conjunto de $\mathrm{m}$ regressores fixos para a curva média da população;

$\phi_{n}\left(t_{i}\right)=$ função de regressão de ordem $\mathrm{k}_{\mathrm{b}}$, que descreve a curva média da população de acordo com o dia de lactação $\left(\mathrm{t}_{\mathrm{i}}\right) ; \phi_{m}\left(t_{i j}\right)=$ função de regressões, que descrevem as trajetórias de cada individuo $\mathrm{j}$, de acordo com o dia da lactação $\left(\mathrm{t}_{i}\right)$, para os efeitos aleatórios genético aditivo e de ambiente permanente; $\alpha_{j m}, \gamma_{j m}=$ são os $m$ regressores aleatórios genético aditivos e de ambiente permanente, para cada animal; $k_{b}, k_{a}$ e $k_{p}=$ são as ordens dos polinômios utilizadas para os efeitos descritos acima; $E_{i j}=$ erro aleatório associado a cada controle $i$ do animal $j$.

Os componentes de variância foram estimados pelo método da Máxima Verossimilhança Restrita (REML), usando um algoritmo livre de derivadas, disponível no pacote WOMBAT (MEYER, 2006). A escolha da ordem das funções de covariância (FC) para ambos efeitos aleatórios (genético aditivo e de ambiente permanente de animal) e da estrutura das variâncias residuais foi feita com base nos resultados de BIGNARDI et al. (2009a), que trabalharam com a mesma característica e parte do banco de dados que foi usado no presente estudo: polinômio de Legendre de sexta ordem para o efeito genético aditivo $\left(\mathrm{k}_{\mathrm{a}}=6\right)$ e de sétima ordem para o efeito de ambiente permanente $\left(\mathrm{k}_{\mathrm{p}}=7\right)$, variâncias residuais heterogêneas, contendo seis classes de variâncias.

\section{RESULTADOS E DISCUSSÃO}

A produção de leite média não se alterou, independente da estrutura de dados empregada, assim como os seus desvios-padrão (Tabela 1). O mesmo foi 
Tabela 1 - Estrutura dos arquivos de dados, número de observações em cada estrutura (OBS), número de grupos de contemporâneos em cada banco de dados (GC), número de touros, mães e animais, produção média por período e número de lactações em cada arquivo estudado.

\begin{tabular}{lllccccc}
\hline Período & OBS & GC & Touros & Mães & Animais & Produção média/kg & Lactações \\
\hline CS & 122.840 & 335 & 228 & 3.202 & 3.202 & 30,16 & 3.202 \\
CM & 30.883 & 335 & 228 & 3.202 & 3.202 & 30,12 \\
CB & 15.837 & 335 & 228 & 3.202 & 3.202 & 30,71 & 3.202 \\
CT & 12.702 & 335 & 228 & 3.202 & 3.202 & 30,07 \\
\hline
\end{tabular}

$\mathrm{CS}=$ semanal; $\mathrm{CM}=$ mensal; $\mathrm{CB}=$ bimestral $\mathrm{CT}=$ trimestral.

constatado quanto ao número de lactações, de grupos de contemporâneas, de touros, vacas e de animais, uma vez que os rebanhos usados no presente estudo possuíam produções de leite diárias ou semanais e a definição dos GC foi mais generalizada.

As estimativas de variâncias genéticas para as PLDC para o banco CS apresentaram tendência semelhante do início $\left(7,71 \mathrm{~kg}^{2}\right)$ até próximo da $20^{\circ}$ semana de lactação $\left(6,81 \mathrm{~kg}^{2}\right)$, aumentando a partir deste ponto até $15,11 \mathrm{~kg}^{2}$ na $43^{\circ}$ semana de lactação. Resultados semelhantes foram relatados por BIGNARDI et al. (2009a), trabalhando com parte deste banco de dados. Os demais arquivos, representando as freqüências mensais, bimestrais e trimestrais apresentaram a mesma tendência, sendo que as magnitudes das variâncias genéticas foram bem próximas às estimadas no banco CS. Além disso, as variâncias genéticas para as PLDC das quatro estruturas também apresentaram maior variabilidade a partir da $30^{\mathrm{a}}$ semana de lactação, sugerindo que a utilização de diferentes frequiências de controles entre os arquivos analisados não acarretou em uma mudança nas tendências e magnitudes das variâncias genéticas entre os arquivos estudados.

Com relação às variâncias de ambiente permanente, todos os bancos analisados apresentaram a mesma tendência, mas com magnitudes iniciais diferentes, com destaque para o banco $\mathrm{CM}$ que apresentou a maior estimativa no início e menor estimativa no final da lactação, compensando a diferença existente nas variâncias residuais (menor no início e maior no final). As variâncias residuais para as PLDC para o arquivo CS foram praticamente constantes durante todo o período analisado. Embora para as demais estruturas a tendência tenha sido semelhante, os valores apresentaram diferenças mais acentuadas em relação aos componentes de variância para os demais efeitos.

As variâncias fenotípicas estimadas para o banco CS apresentaram, inicialmente, um valor de $35,79 \mathrm{~kg}^{2}$, oscilando um pouco até a $13^{\mathrm{a}}$ semana e aumentando gradativamente até a $43^{\mathrm{a}}$ semana de lactação. Tendência semelhante, mas com magnitudes inferiores foram descritas por BIGNARDI et al. (2009a).

Para todos os bancos de dados analisados as estimativas de herdabilidade para as PLDC apresentaram pequenas oscilações, e tendências de crescimento nas estimativas do início para o final da lactação. Para o arquivo CS, as herdabilidades variaram de 0,17 a 0,33 . A primeira metade da lactação apresentou menores valores de herdabilidade que a segunda metade, sendo que a maior estimativa ocorreu na $32^{\mathrm{a}}$ semana. Valores equivalentes foram relatados por COSTA et al. (2008) e MELO et al. (2005) trabalhando com primeiras lactações de vacas Holandesas no Brasil.

As herdabilidades estimadas para as PLDC dos bancos CM e CB foram muito próximas das estimadas para o banco $\mathrm{CS}$, em tendência e magnitude. Para o arquivo $\mathrm{CB}$ as herdabilidades variaram de 0,19 na fase inicial da lactação até 0,29 na fase final. Para o arquivo CM estas estimativas variaram de 0,19 a 0,29 .

Independente da estrutura, todos os bancos analisados apresentaram maiores estimativas de herdabilidade para as PLDC na segunda metade da lactação, a partir do $30^{\circ}$ controle, assim como observado para as variâncias genéticas. Assim, é possível obter maior eficiência de seleção para maximizar o ganho genético para a produção de leite nessa fase.

As estimativas de correlações genéticas entre as PLDC variaram de $-0,27$ a 0,99 para a estrutura CS, de $-0,08$ a 0,99 para CM, de -0,02 a 0,98 para CB e de -0,64 a 0,61 para o banco trimestral (CT). As correlações genéticas negativas sempre ocorreram entre as produções do início com as produções do final da lactação. Para todas as estruturas estudadas, as superfícies mostraram que ocorreram oscilações 
nas estimativas de correlação genética, quando o desejável seriam superfícies mais planas, sem grandes oscilações.

De maneira geral, as estimativas foram maiores quanto mais próximos os controles leiteiros, diminuindo à medida que aumentou a distância entre os mesmos, com exceção do banco CT, cuja amplitude nas estimativas de correlações genéticas foi maior, ocorrendo também menores estimativas de correlações entre as PLDC do meio da lactação. Isso pode ser explicado pela distância maior entre os controles e ao menor número de controles analisados neste arquivo de dados, sugerindo interferência da estrutura de dados nas estimativas do banco CT. As altas magnitudes das correlações genéticas estimadas entre as PLDC indicam que a seleção para a produção em qualquer período levaria a um ganho genético correlacionado na produção de leite nos demais períodos, considerando as estruturas $\mathrm{CS}, \mathrm{CM}$ e $\mathrm{CB}$. Para o arquivo CT, isso não poderia ser afirmado.

As correlações fenotípicas, assim como as genéticas, apresentaram tendências e superfícies semelhantes para as diferentes estruturas testadas. Da mesma maneira, como o arquivo CT possuía menor número de informações, a superfície apresentou oscilações mais visíveis que para as demais estruturas. De maneira geral, as correlações fenotípicas foram maiores entre os controles adjacentes e apresentaram menores magnitudes que as correlações genéticas, sendo as magnitudes, próximas às encontradas por COBUCI et al. (2005), FERREIRA et al. (2003) e MELO et al. (2005). As correlações de ambiente permanente para os diferentes arquivos de dados analisados apresentaram superfícies e tendências muito próximas, mantendo o mesmo comportamento das correlações anteriores.

As correlações de Spearman e Pearson entre os valores genéticos preditos para a produção de leite acumulada em 305 dias (MRA305) apresentaram magnitudes positivas e de médias a altas ao comparar os diferentes arquivos de dados e categorias de animais selecionados (Tabela 2). Os MRA305 foram obtidos para cada animal por meio da somatória dos seus valores genéticos diários preditos pela curva genética de cada animal. Foi observado que à medida em que a frequiência dos dados diminuiu, houve redução na correlação entre os valore genéticos (VG), independente da categoria dos animais selecionados. Quando 5\% dos melhores touros foram selecionados com base no MRA305, as correlações foram maiores que para as demais categorias de animais, provavelmente porque o número de filhas por touro pode ter contribuído para melhorar a predição da
Tabela 2 - Correlação de Spearman (acima da diagonal) e de Pearson (abaixo da diagonal) entre as diferentes estruturas de dados, para 5\% de touros, vacas e todos os animais selecionados, com base no valor genético para MRA305.

\begin{tabular}{|c|c|c|c|c|}
\hline Arquivos & $\mathrm{CS}$ & $\mathrm{CM}$ & $\mathrm{CB}$ & CT \\
\hline \multicolumn{5}{|c|}{ 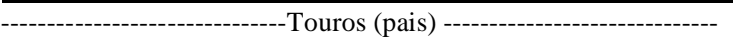 } \\
\hline $\mathrm{CS}$ & - & 0,86 & 0,85 & 0,83 \\
\hline $\mathrm{CM}$ & 0,90 & - & 0,89 & 0,85 \\
\hline $\mathrm{CB}$ & 0,87 & 0,91 & - & 0,82 \\
\hline $\mathrm{CT}$ & 0,88 & 0,89 & 0,86 & - \\
\hline \multicolumn{5}{|c|}{ - } \\
\hline $\mathrm{CS}$ & - & 0,70 & 0,53 & 0,44 \\
\hline $\mathrm{CM}$ & 0,72 & - & 0,77 & 0,65 \\
\hline $\mathrm{CB}$ & 0,55 & 0,78 & - & 0,69 \\
\hline $\mathrm{CT}$ & 0,47 & 0,70 & 0,72 & - \\
\hline \multicolumn{5}{|c|}{ Todos os Animais com dados- } \\
\hline CS & - & 0,74 & 0,59 & 0,50 \\
\hline $\mathrm{CM}$ & 0,74 & - & 0,78 & 0,68 \\
\hline $\mathrm{CB}$ & 0,61 & 0,79 & - & 0,72 \\
\hline $\mathrm{CT}$ & 0,51 & 0,71 & 0,74 & - \\
\hline
\end{tabular}

$\mathrm{CS}=$ semanal; $\mathrm{CM}=$ mensal; $\mathrm{CB}=$ bimestral $\mathrm{CT}=$ trimestral.

curva de lactação genética para os touros e assim, a estrutura das informações da PLDC teria menor impacto.

As correlações entre os valores genéticos de vacas para os quatro arquivos de dados estudados apresentaram valores menores que os observados para os 5\% melhores touros, sendo todas as correlações inferiores a $80 \%$ e havendo uma maior discrepância entre os diferentes arquivos, em relação ao arquivo base de comparação, que foi o banco semanal (Tabela 2).

Para os touros, por exemplo, não houve grande diferença das correlações de posto entre as estruturas semanal com a mensal (86\%), a bimestral $(85 \%)$ e a trimestral $(83 \%)$. Já para as demais categorias, indicando vacas e todos os animais, ocorreram maiores discrepâncias. Estes resultados demonstram que existem diferenças nas classificações dos animais, ou seja, pouca semelhança entre estes arquivos de dados, e estas diferenças são maiores para vacas e todos os animais, sugerindo que o número de informações por animal (por exemplo, o número de filhas por touro) pode amenizar o problema de um controle leiteiro mais espaçado.

$\mathrm{Na}$ tabela 3 estão apresentadas as classificações dos 5, 10 e 20\% melhores touros, com base nos valores genéticos para MRA305, para os quatro bancos de dados. Pôde-se observar relativas coincidências entre os arquivos. Houve bastante 
Tabela 3 - Arquivos, touros em comum nos diferentes arquivos quando selecionados para MRA305 no arquivo semanal, considerando-se os níveis de 5, 10 e $20 \%$ de touros selecionados.

\begin{tabular}{|c|c|c|c|}
\hline \multirow{2}{*}{ Arquivos } & 5\% Melhores & 10\% Melhores & $20 \%$ Melhores \\
\hline & 11 touros & 23 touros & 45 touros \\
\hline $\mathrm{CM}$ & $9(82 \%)$ & $15(65 \%)$ & $31(69 \%)$ \\
\hline $\mathrm{CB}$ & $8(73 \%)$ & $15(65 \%)$ & $33(73 \%)$ \\
\hline $\mathrm{CT}$ & $8(73 \%)$ & $15(65 \%)$ & $30(67 \%)$ \\
\hline
\end{tabular}

$\mathrm{CS}=$ semanal; $\mathrm{CM}=$ mensal; $\mathrm{CB}=$ bimestral; $\mathrm{CT}=$ trimestral.

similaridade entre as classificações dos touros para os arquivos CM, CB e CT, entretanto, algumas mudanças ocorreriam na classificação em relação ao arquivo CS. Para os 5\% dos melhores touros dos arquivos, tendo como base o valor genético (MRA305) em CS, o arquivo CM apresentou nove touros em comum selecionados (82\% do total). Situação semelhante ocorreu com as estruturas CB e CT. Para os demais percentuais de animais selecionados, ou seja, para os $10 \%$ e $20 \%$ melhores touros, as coincidências foram menores, em torno de $65 \%$ e $69 \%$ do total, respectivamente. Embora as coincidências sejam razoáveis, os percentuais indicam que ocorreram divergências na escolha dos melhores touros e, conseqüentemente, na classificação dos mesmos.

SHAHRBABAK (1997), comparando diferentes estruturas de dados com frequiências alternadas entre controles de animais da raça Holandesa oriundos do Irã, quando foram comparados controles mensais (arquivo controle), bimestrais e trimestrais, o autor obteve sensíveis mudanças na classificação dos touros, quando comparou o arquivo mensal (arquivo controle) com os demais arquivos. $\mathrm{O}$ autor observou boas similaridades entre os arquivos de dados e obteve também altas correlações entre os VG de touros para produção de leite entre os arquivos bimestral e arquivo controle.

No presente estudo foram observadas similaridades entre as estimativas de variâncias e herdabilidades para PLDC nos diversos arquivos de dados analisados, porém ao se comparar as correlações de posto entre os valores genéticos de touros, mãe e animais do arquivo semanal (arquivo controle) com os demais arquivos de dados foi possível verificar pequenas diferenças nas classificações dos animais quando foram comparados os arquivos CS e CT, podendo assim ocorrer a seleção de diferentes animais ao se utilizar o arquivo de dados trimestral. Logo a utilização do arquivo CB e CM seriam alternativas indicadas, uma vez que estes apresentaram boas semelhanças e similaridades com o arquivo referência.

Em geral, no Brasil, o sistema de controle leiteiro oficial adota o intervalo mensal entre os controles em média. $\mathrm{O}$ presente estudo pode auxiliar na busca de alternativas no controle leiteiro, principalmente em lugares onde o acesso do controlador é mais difícil. O ideal seria a realização do controle mensal, mas não haveria grandes prejuízos nas avaliações genéticas de reprodutores aplicando modelos de regressão aleatória, no caso de o mesmo ser realizado bimestralmente, principalmente se isto acarretasse em maior número de animais sendo controlados.

\section{CONCLUSÃO}

As estruturas de controle semanais, mensais e bimestrais não apresentaram grandes diferenças entre seus parâmetros genéticos, entretanto o banco com freqüências de controle trimestral apresentou estimativas dos seus parâmetros genéticos diferentes dos demais bancos, devido ao maior intervalo entre os controles.

As predições de valores genéticos e suas correlações entre os arquivos CS e CM apresentaram maiores semelhanças e similaridades quando comparados às demais estruturas, entretanto houve mudanças nas classificações, o que pode acarretar na seleção de diferentes animais, principalmente para mães e animais com dados.

A utilização dos arquivos de controle CM e CB pode ser indicada para programas de controle leiteiro, uma vez que estes apresentaram grandes similaridades com o arquivo semanal.

\section{REFERÊNCIAS}

ALBUQUERQUE, L.G.; MEYER, K. Estimates of covariance functions for growth of Nelore cattle applying a parametric correlation function to model within-animal correlations. Livestock Production Science, v.93, n.4, p.213-222, 2005.

BIGNARDI, A.B.; EL FARO, L.; ALBUQUERQUE, L.G.; CARDOSO, V.L.; MACHADO, P.F. Random regression models to estimate test-day milk yield genetic parameters Holstein cows in southeastern Brazil. Livestock Science, v.123, p.1-7, 2009a.

BIGNARDI, A.B.; EL FARO, L.; ALBUQUERQUE, L.G.; CARDOSO, V.L.; MACHADO, P.F. Parametric correlation functions to model the structure of permanent environmental (co) variances in milk yield random regression models. Journal Dairy Science, v.92, p.4634-4640, 2009b. 
COBUCI, J. A.; EUCLYDES, R. F.; LOPES, P. S.; et al. Estimation of genetic parameters for test-day milk yield in Holstein cows using a rondam regression model. Genetics and Molecular Biology, v.28, n.1, p.75-83, 2005.

COSTA, C. N.; MELO, C. M. R.; PAKER, I. U. Genetic parameters for test milk yield of first lactation Holstein cows estimated by radom regression using Legendre polynomials. Revista Brasileira de Zootecnia, v.37 n.4 p.602-608, 2008.

EL FARO, L.; ALBUQUERQUE, L.G. Utilizando Modelos de Regressão Aleatória para produção de leite no dia do controle, com diferentes estruturas de variâncias residuais. Revista Brasileira de Zootecnia, v.32, n.5, p.1104-1113, 2003.

FERREIRA, W. J.; TEIXEIRA, N. M.; EUCLUDES, R. F. Avaliação genética de bovinos da raça Holandesa usando produção de leite no dia do controle. Revista Brasileira de Zootecnia v.32, n.2, p.295-303, 2003.

MELO, C. M. R; GONÇALVES, T. M; MARTINEZ, M. L; VERNEQUE, R. S; OLIVEIRA, A. I. G; FREITAS, R. T. F. Avaliação genética de touros usando produção em lactação completas ou parciais projetadas. 2. Correlação e coincidência de ordem de "rank". Revista Brasileira de Zootecnia, v. 29, n.3, p.715-719, 2000.

MELO, C. M. R; PACKER, I. U. COSTA, C. N; MACHDO, P. F. Parâmetros genéticos para as produções de leite no dia do controle e da primeira lactação de vacas da raça Holandesa. Revista Brasileira de Zootecnia, v. 34, n.3, p.796-806, 2005.

MEYER, K. Modeling 'reapeted' records: covariance functions and random regression models to analyze animal breeding data. In: WORLD CONGRESS ON GENETICS APPLIED TO LIVESTOCK PRODUCTION, 6. 1998a, Armidale, Proceedings... Armidale, 1998b. v.25, n.6, p.517-520, 1998a.

MEYER, K. Estimating covariance functions for longitudinal data using a random regression model. Genetic Selection Evolution, v.30, p.221-240, 1998b.

MEYER, K. Estimates of direct and maternal covariance functions for growth of Australian beef calves from birth to weaning. Genetic Selection Evolution, v.33, p.487-514, 2001

MEYER, K. WOMBAT - Digging deep for quantitative genetic analyses by restricted maximum likelihood" In: WORLD CONGRESS ON GENETICS APPLIED TO LIVESTOCK PRODUCTION, 8, 2006, Belo Horizonte. Proceedings... Belo Horizonte, 2006. I CD-ROM.

SHAHRBABAK, M.M. Feasibility of random regression models for Iranian Holstein testday records. Thesis (PHD) - University of Guelph, Guelph, Canadá. P.138, 1997. 